\title{
Ibrutinib Therapy for Chronic Lymphocytic Leukemia Complicated with Secondary Serous Adenocarcinoma of the Peritoneum
}

\author{
Bartosz PULA, Krzysztof JAMROZIAK \\ Institute of Hematology and Transfusion Medicine, Department of Hematology, Warsaw, POLAND
}

To the editor,

Chronic lymphocytic leukemia (CLL), the most common leukemia in Europe and the USA, affects predominantly elderly patients who typically present with coexisting medical health problems which may independently impact survival. A prospective cohort study identified CLL progression $(46 \%)$ as the main cause of death; however, two other major reasons of patients' demise were comorbid health conditions $(27 \%)$ and secondary cancers $(19 \%){ }^{1}$

In the era of the treatment of CLL with alkylating drugs and purine nucleoside analogs an increased risk of secondary tumours including skin cancers melanoma, glioblastoma and cancers of the lungs, larynx, urinary bladder and stomach have been reported that is likely due to the immunocompromising nature and the treatment. However, less is known regarding the susceptibility to secondary tumours in CLL patients treated with modern nonchemotherapy signaling pathways inhibitors, such as ibrutinib, idelalisib and venetoclax.

Ibrutinib, a covalent Bruton's tyrosine kinase (BTK) inhibitor, achieved remarkable results in CLL and significantly improved patients' outcomes including those with high-risk features, both in controlled clinical trials as well as in real- world practice setting. ${ }^{2-4}$ The drug is well tolerated, however, a spectrum of characteristic side effects such as atrial fibrillation, elevated blood pressure, bleeding diathesis and lung infections has been described. ${ }^{5-7}$ Interestingly, up to date reports addressing ibrutinib impact on development of secondary malignancies remain scarce. Furthermore, further management of a CLL patient with a diagnosis of secondary cancer established while on ibrutinib has not been discussed by international guidelines. ${ }^{5-7}$ Therefore, we describe our experience with CLL patient developing primary serous adenocarcinoma of the peritoneum under ibrutinib therapy.

A 63-year old female patient was referred to the Department of Hematology of the Institute of Hematology and Transfusion Medicine in April 2014 with a history of leukocytosis and lymphocytosis that had been observed in occasional blood tests since 2010. Past medical history was characterized by elevated blood pressure, nodular goiter and appendectomy. At that moment blood morphology revealed whole blood cell count (WBC) $89 \mathrm{G} / \mathrm{L}$ with blood smear showing $40 \%$ lymphocytes and 54\% prolymphocytes, hemoglobin (HGB) 12.3 $\mathrm{g} / \mathrm{dL}$ and platelet count (PLT) $76 \mathrm{G} / \mathrm{L}$. Peripheral blood immunophenotyping confirmed the diagnosis of CLL. 
An elevated lactate dehydrogenase (LDH) activity $(545 \mathrm{U} / \mathrm{L})$ and total bilirubin $(1.25 \mathrm{mg} / \mathrm{dl})$ was shown, however no signs of hemolysis could be noted (normal level of reticulocytes and haptoglobin, negative Coombs test). Ultrasonographical examination revealed enlarged peripheral lymph nodes (44 mm left axillary region, $2-3 \mathrm{~cm}$ in other regions) as well as enlarged abdominal lymph nodes (up to $31 \mathrm{~mm}$ ), spleen and liver were within normal limits. Bone marrow smear analysis showed an $88 \%$ lymphocyte infiltrate, whereas upon histopathological examination of the trephine biopsy a CLL infiltrate reaching $95 \%$ was recognized. Following the diagnostic evaluation her disease was staged Rai IV due to mild stable thrombocytopenia of approx. $80 \mathrm{G} / 1$. The decision on treatment initiation was temporarily withheld, but in May 2014 her platelet count decreased significantly with associated symptoms of hemorrhagic diathesis including epistaxis and muco-cutaneous ecchymoses. Since no other signs of CLL progression were present secondary immune thrombocytopenia was diagnosed. Steroid therapy (short course of high-dose dexamethasone) was administered; however, with no platelet response. Subsequently, six courses of rituximab, cyclophosphamide and dexamethasone (RCD) immunochemotherapy were given between May and November 2014. Following this therapy blood counts including platelets returned to normal, but due to persistent lymphadenopathy response was assessed as partial remission. In March 2015 rapid enlargement of lymph nodes of all groups was noted. At that time no significant aberration in peripheral blood have been noted, however ultrasonography of peripheral lymph nodes confirmed disease progression with axillar lymph nodes being the largest and reaching 44x26 $\mathrm{mm}$. No splenomegaly or hepatomegaly was noted. Bone marrow cytology showed predominant lymphocytic (39.8\%) and prolymphocytic (30.8\%) infiltration, whereas trephine biopsy showed $70 \%$ CLL/SLL cells infiltrate. Additionally, fluorescent in situ hybridization (FISH) of peripheral blood lymphocytes was performed revealing 11q (ATM gene) deletion, whereas no other tested abnormalities including deletion $17 \mathrm{p}$, deletion $13 \mathrm{q} 14$ or trisomy 12 were identified.
Second line therapy with ibrutinib $420 \mathrm{mg}$ daily was initiated in frame of worldwide compassionate use Named Patient Program of Janssen-Cilag pharmaceutical company. ${ }^{4,7}$ Following 2 months of such treatment an increase of lymphocytosis with concomitant reduction of lymph node size was observed, and a partial response with lymphocytosis (PR-L) was recognized. Ibrutinib therapy was reasonably well tolerated. Among early ibrutinib adverse events worsening of preexisting hypertension that required dose increase of angiotensinconverting-enzyme (ACE) inhibitor and ecchymose with normal platelet counts were observed. As of November 2016, abdomen ultrasonography revealed almost complete resolution of lymphadenopathy with only a few persisting lymph nodes of borderline size $(17 \times 6 \mathrm{~mm})$ near the liver hilum, and the therapy of ibrutinib was continued in a standard dose.

In January 2017 the patient started to complain of pain in the lower part of the abdomen, dysuria and constipations with narrow stools. The ultrasonography of the abdomen performed in February 2017 revealed a large heterogenous and well vasculated tumor mass of $108 \times 35 \mathrm{~mm}$ localized in the hypogastric region. Subsequent abdominal computed tomography (CT) confirmed presence of a large bulky mass measuring $163 \times 50 \times 90 \mathrm{~mm}$ that extended from the abdominal wall, through the omentum to the upper wall of the urinary bladder and left ovary. The patient was qualified to surgical intervention with a suspicion of advanced metastatic cancer originating from the left ovary. Ibrutinib therapy was withheld before the surgical procedure according to characteristics of the medicinal product. At the time of ibrutinib interruption patient's blood count remained stable (WBC 11.05 G/l, HGB 12.2 g/dl, PLT 302 G/l), with largest lymph nodes measuring $14 \times 9 \mathrm{~mm}$ in the abdomen and $15 \times 14 \mathrm{~mm}$ in the thoracic cavity (maintained PR-L response). On 8th March 2019 the procedure of total hysterectomy through medial laparotomy was performed. Intraoperative histopathological examination revealed serous carcinomatous infiltrates in sampled peritoneal nodules, omentum and right fallopian tube with no pathological cells being detected in uterus, ovaries and left fallopian tubes. Histopathological examination confirmed the pres- 
ence of poorly differentiated serous cancerous cells (G3 differentiation) expressing WT1 antigen, whereas no expression of podoplanin, cytokeratin 20 (CK20) and p53 protein could be noted. A diagnosis of stage IV primary serous carcinoma of the peritoneum was established.

Due to advanced tumour stage and the underlying CLL the decision of starting palliative carboplatin monotherapy was made in the Daily Oncology Department of the Magodent Hospital in Warsaw in April 2017. Eight carboplatin cycles were administered until October 2017 with partial regression of infiltrative masses seen in the control CT scan performed after. In December 2017 ibrutinib was reinitiated with good tolerance and stable CLL remission (PR-L). However, in February 2018 the patient underwent thoracentesis due to right pleural effusion provoking dyspnea and cough. Fluid analysis revealed presence of metastatic atypical cells, and right pleural pleurodesis was done in March 2018. Due to symptoms of cancer dissemination (pleural effusion, abdominal lymphadenopathy and liver metastases) a new line of palliative chemotherapy with paclitaxel was administered. Altogether the patient received four cycles of paclitaxel therapy, while ibrutinib treatment was continued. The simultaneous administration of both compounds was well tolerated with grade 2 anemia being the only hematological adverse event observed during the treatment. Nevertheless, due to progression of serous carcinoma since August 2018 the therapy was changed to four cycles of liposomal doxorubicin. However, this time the tolerance of the treatment was poor with fatigue, loss of appetite, grade 2 anemia, bleeding diathesis and grade 2 polyneuropathy. For this reason, the dose of ibrutinib was reduced to $280 \mathrm{mg}$ daily. Despite the continuous administration of ibrutinib and good control of the underlying CLL, patient died in March 2019 due to cancer progression, while underlying CLL remained well controlled by the ibrutinib monotherapy.

The case presented here questions the role of a prolonged therapy with ibrutinib as the potential factor facilitating development of a secondary serous adenocarcinoma of the peritoneum with aggressive course. It needs to be underlined that the patient was well monitored during the CLL treatment, and the abdomen ultrasonography performed only two months before tumour diagnosis showed only minimal abdominal lymphadenopathy, probably in the course of CLL.

To our best knowledge this is the first report of this tumor type in a patient treated with ibrutinib. ${ }^{5-7}$ Although the relationship of ibrutinib and development or acceleration of the serous adenocarcinoma of the peritoneum is uncertain, it needs to be considered that the drug mechanism of action significantly interferes with immune tolerance. ${ }^{8}$ Furthermore, although ibrutinib has not yet been shown to be linked to increased risk of secondary cancer occurrence, the recent long-term observation of patients from the RESONATE trial revealed that non-melanoma skin cancers occurred in $15 \%$ of ibrutinib treated patients. Whether these effects could be ascribed to ibrutinib itself is a matter of debate, because all of the patients qualified for the trial underwent previous cytotoxic treatment. ${ }^{2}$

Another important issue is the possibility of continuing ibrutinib in a CLL patient that is receiving anti-cancer treatment for another tumour. It is well known that ibrutinib needs to be continued indefinitely as the interruption of the therapy leads to rapid CLL progression. On the other hand, ibrutinib has many metabolic interactions with numerous drugs as well as some side effects that may increase the risk of cytotoxic therapy (e.g. inhibition of platelet activity). In our case, it was decided to reinitiate ibrutinib treatment to prevent CLL relapse after control of adenocarcinoma had been achieved with first line carboplatin therapy. The main reason for such decision was the fact that in contrast to standard immunotherapy BTK-inhibitor treatment rarely results in cytopenia what enables the continuation of cytotoxic therapy against solid tumour, thus we judged such concomitant therapy relatively safe. Secondly, there is increasing evidence from preclinical data indicating the role of BTK in progression of prostate, ovarian, gastric and breast cancer models and clinical trials assessing ibrutinib efficacy in several solid tumours are underway. ${ }^{9} 10$ Taking this into consideration coadministration of ibrutinib with other cytostatic agents could potentially have additive anti-cancer 
and anti-leukemic effect and target both diseases. In this case combining ibrutinib for CLL and palliative cytotoxic chemotherapy for serous adenocrcinoma resulted rather safe and the patient survived for additional 2 years from the diagnosis of aggressive secondary tumour. However, the impact of such combination on clinical course of both diseases is difficult to estimate.

In conclusion, ibrutinib therapy may be safely continued during cytostatic treatment of a secondary CLL malignancy, however its coadministration should be made with caution including the specific types of adverse events of administered drugs. Considering the BTK inhibitors' potential cardiotoxicity and hemostasis one could expect their potentiation with use of standard cytotoxic agents, however none of this was observed. To note, the efficacy of simultaneous treatment with ibrutinib and other cytostatic drugs is so far poorly recognized due to lack of clinical trials and sparse real-world patient data. Furthermore, clinical guidelines and recommendations addressing this issue should developed.

\section{REFERENCES}

1. Strati P, Parikh SA, Chaffee KG, et al. Relationship between co-morbidities at diagnosis, survival and ultimate cause of death in patients with chronic lymphocytic leukaemia (CLL): a prospective cohort study. Br J Haematol 178: 394-402, 2017.

2. Byrd JC, Hillmen P, O'Brien S, et al. Long-term follow-up of the RESONATE phase 3 trial of ibrutinib vs ofatumumab. Blood 133: 2031-2042, 2019.

3. Burger JA, Tedeschi A, Barr PM, et al. Ibrutinib as Initial Therapy for Patients with Chronic Lymphocytic Leukemia. N Engl J Med 373: 2425-2437, 2015.
4. Pula B, Budziszewska BK, Rybka J, et al. Comparable efficacy of Idelalisib plus Rituximab and Ibrutinib in relapsed/ refractory chronic lymphocytic leukemia: A retrospective case matched study of the Polish Adult Leukemia Group (PALG). Anticancer Res 38: 3025-3030, 2018.

5. Jamroziak K, Pula B, Walewski J. Current treatment of chronic lymphocytic leukemia. Curr Treat Options Oncol 18: 5, 2017.

6. Maddocks KJ, Ruppert AS, Lozanski G, et al. Etiology of Ibrutinib therapy discontinuation and outcomes in patients with chronic lymphocytic leukemia. JAMA Oncol 1: 80-87, 2015.

7. Iskierka-Jazdzewska E, Pula B, Szeremet A, et al. Ibrutinib discontinuation in patients with relapsed or refractory chronic lymphocytic leukemia treated in a compassionate use program: A report from the Polish Adult Leukemia Study Group (PALG). Adv Clin Exp Med 28: 1051-1057, 2019.

8. Ryan CE, Sahaf B, Logan AC, et al. Ibrutinib efficacy and tolerability in patients with relapsed chronic lymphocytic leukemia following allogeneic HCT. Blood 128: 2899-2908, 2016.

9. Molina-Cerrillo J, Alonso-Gordoa T, Gajate P, Grande E. Bruton's tyrosine kinase (BTK) as a promising target in solid tumors. Cancer Treat Rev 58: 41-50, 2017.

10. Pal Singh S, Dammeijer F, Hendriks RW. Role of Bruton's tyrosine kinase in B cells and malignancies. Mol Cancer 17: 57, 2018.

\section{Correspondence:}

Bartosz PULA, MD, PhD.

Department of Hematology

Institute of Hematology and Transfusion Medicine

Indiry Gandhi Str. 14, 02-776

WARSAW / POLAND

Tel: +48223496334

Fax: +48 223496335

e-mail: bartosz.pula@gmail.com 